\title{
LA MÉDECINE ET LE NUCLÉAIRE
}

\section{Introduction}

\author{
par Maurice TUBIANA \\ Professeur à la Faculté de Médecine de Paris; \\ Chef du Département des Radiations de l'Institut Gustave Roussy
}

\begin{abstract}
Les rayonnements ionisants sont, depuis la fin du siècle dernier, progressivement devenus un des éléments les plus importants de l'environnement médical. Quelques mois à peine après la découverte des rayons $X$ par Röntgen, des médecins de tous les pays d'Europe reproduisaient ses expériences et utilisaient ces mystérieux rayons $\mathrm{X}$ pour étudier le squelette humain. La vitesse avec laquelle les travaux de Röntgen ont été connus et reproduits donne une idée de l'extraordinaire rapidité de la diffusion des nouvelles dans la petite communauté scientifique de la fin du $\mathrm{XIX}^{\ominus}$ siècle.
\end{abstract}

\section{Radiodiagnostic et radiothérapie}

Les découvertes de Becquerel et Pierre Curie n'ont pas eu un moindre retentissement bien qu'elles aient initialement moins directement concerné les milieux médicaux. Cependant, l'observation par Becquerel d'un érythème cutané en regard du gousset du gilet dans lequel il avait transporté des radioéléments est la première observation de radiobiologie et a fait l'objet d'une note à l'Académie des Sciences, signée conjointement avec Pierre Curie après que celui-ci ait vérifié la réalité du phénomène en mettant à son tour les mêmes radioéléments dans son gilet.

Radiodiagnostic et radiothérapie se sont depuis rapidement développés. Dès l'entre-deux-guerres, ils avaient acquis une place essentielle dans le diagnostic et le traitement de nombreuses maladies.

De simples "photographes", les radiodiagnosticiens sont progressivement devenus des spécialistes indispensables pour effectuer et, surtout, interpréter les innombrables types d'examens radiologiques qu'a rendus possibles l'utilisation des produits de contraste utilisés pour visualiser les vaisseaux ou les cavités qui ne se distinguent pas sur les clichés sans préparation (reins, voies biliaires, tube digestif, bronches, cavités du cerveau, artères, veines et voies lymphatiques, etc.). Depuis quelques années, le traitement sur ordinateur des informations obtenues en balayant sous différents angles avec un fin pinceau de rayons $\mathrm{X}$ la région de l'organisme étudié permet d'établir les contours des zones de différentes densités à l'intérieur de l'organisme. Le tomo-densito- mètre fournit ainsi de véritables coupes anatomiques de l'organisme et ces nouveaux instruments, malgré leur prix élevé (trois à cinq millions de francs), sont en train de bouleverser la pratique du radiodiagnostic.

La radiothérapie a subi, elle, une grande mutation il y a une vingtaine d'années avec le remplacement des générateurs de rayons $X$ par le cobalt radioactif et les grands accélérateurs (Bétatron et accélérateur linéaire) grâce auxquels on peut utiliser des photons de haute énergie, beaucoup plus pénétrants et qui permettent donc de délivrer des doses élevées à la tumeur tout en réduisant l'irradiation des tissus sains. Parallèlement, le radium était remplacé par des isotopes radioactifs artificiels dont l'usage est plus souple et se prête mieux aux diverses modalités d'implantation radioactive. Les ordinateurs, en accroissant la rapidité et la précision des calculs établissant la répartition des doses dans l'organisme ont également contribué aux progrès récents de la radiothérapie.

\section{La médecine nucléaire}

La troisième grande discipline médicale basée sur l'emploi des radiations ionisantes est la médecine nucléaire. Née à la fin de la dernière guerre, elle utilise les isotopes radioactifs, soit comme traceurs pour étudier un métabolisme ou l'activité fonctionnelle d'un organe soit comme agent de visualisation d'un tissu en scintigraphie, soit enfin comme agent thérapeutique en disposant la source radioactive au sein même du tissu à traiter. Le remplacement des compteurs de Geiger par les compteurs à scintillation avait, il y a vingt-cinq ans, constitué une première étape technologique importante. II y a dix ans, l'introduction des caméras à scintillation, qu permettent en quelques minutes de visualiser la répartition d'un radioélément dans l'organisme, avait représenté une nouvelle étape technologique. Enfin, tout récemment, le traitement sur ordinateur des informations fournies par les caméras à scintillation a permis un nouveau progrès dont les possibilités n'ont pas encore été complètement exploitées.

Ces trois disciplines ont des préoccupations communes: la radiobiologie, c'est-à-dire l'étude de l'effet des radia- tions sur les tissus, la radioprotection, c'est-à-dire l'étude des effets pathogènes des radiations sur l'homme et des moyens propres à réduire les effets délétères et à maintenir l'irradiation dans des limites acceptables.

\section{Le coût et le bénéfice}

Tout acte médical, d'une simple piqûre intraveineuse à l'administration d'un cachet d'aspirine, comporte un risque faible mais non nul et qui peut être calculé. Toute décision médicale repose sur une analyse du coût et du bénéfice des divers actes possibles, voire d'une abstention thérapeutique.

Ces analyses étaient presque toujours intuitives jusqu'à la dernière guerre. Un des grands progrès de la médecine depuis trente ans a été de quantifier progressivement les bénéfices grâce aux données statistiques et aux essais thérapeutiques. Mais l'évaluation du coût (risque de complication, danger d'effet secondaire, risque génétique, etc.) reste généralement assez imprécise. C'est sans doute dans le domaine des radiations ionisantes que le plus grand effort a été fait pour quantifier ce "coût", ce qui permet de chiffrer les inconvénients potentiels d'un examen radiologique ou isotopique. Ceci a été rendu possible par l'accroissement des connaissances radiobiologiques qui a lui-même été stimulé par le développement de l'énergie atomique. Cet effort commence à servir de modèle pour des recherches similaires sur la toxicité et les effets génétiques de divers produits chimiques et notamment de nombreux médicaments.

Mais, paradoxalement, l'avance acquise par les radiobiologistes en estimant le risque des radiations ionisantes vaut à celles-ci un réputation de danger qui suscite bien des inquiétudes et amène notamment aux Etats-Unis, certains malades à refuser des examens qui pourraient leur être utiles ou à n'accepter ceux-ci qu'avec réticence. C'est pourquoi, sans nier les dangers des radiations et tout en cherchant des méthodes susceptibles de réduire l'irradiation des malades en radiodiagnostic et en médecine nucléaire, un effort d'information est nécessaire, effort qui rejoint d'ailleurs celui effectué dans le domaine de l'utilisation pacifique de l'énergie nucléaire. 